\title{
Privacy on the road? Evaluating German consumers' intention to use connected cars
}

\author{
Christoph Buck*, Riccardo Reith \\ Centre for Future Enterprise, QUT Business School \\ Queensland University of Technology \\ 2 George St, Brisbane City QLD 4000, Australia \\ Email: christoph.buck@qut.edu.au \\ University of Bayreuth \\ Universitätsstraße 30, 95448 Bayreuth, Germany \\ Email: riccardo.reith@uni-bayreuth.de \\ *Corresponding author
}

\begin{abstract}
Cars are developing into networked systems and digital entities. In order to improve service quality, enable new value propositions, and achieve a better driving experience, connected cars increasingly utilize the data and devices of their occupants. This situation is completely new for consumers, as cars have not been viewed as data collecting devices of highly personal information so far. These novel developments raise the question whether consumers realize and are concerned about the disclosure of sensitive personal data while using connected cars. Research in the field of privacy indicates that consumers value their privacy and that privacy concerns induce privacy behaviour. Therefore, this study aims to empirically validate a research model which considers privacy concerns as a crucial construct in predicting the intention to use connected cars. The results of our investigation enhance the understanding of consumers' privacy concerns in connected cars and will help car manufacturers to adequately address these issues within their market strategy.
\end{abstract}

Keywords: Connected cars, privacy concerns, privacy calculus, consumer acceptance, acceptance model

Reference to this paper should be made as follows: Buck, C., Reith, R. (2020): Privacy on the road? Evaluating German consumers' intention to use connected cars, Int. J. Automotive Technology Management,

Biographical notes: Christoph Buck is a post-doctoral fellow at the Centre for Future Enterprise at the Queensland University of Technology. His research interests include digital transformation, innovation systems and information privacy.

Riccardo Reith is a doctoral student at the University of Bayreuth. In his research he focusses on information privacy and technology acceptance. 